\title{
Longitudinal structure function from the parton parameterization
}

\author{
B.Rezae:* and G.R.Boroun \\ Physics Department, Razi University, Kermanshah 67149, Iran
}

(Dated: June 23, 2020)

\begin{abstract}
We present a certain theoretical model to describe data based on the DGLAP evolution equations at low values of $x$. This model is based on a hard poemron exchange in the next-to-next-to-leading order of the perturbative theory. The behavior of the DIS cross section ratio $R\left(x, Q^{2}\right)$ and $F_{L}\left(x, Q^{2}\right) / F_{2}\left(x, Q^{2}\right)$ is studied and compared with the experimental data. These behaviors are controlled by the color dipole model bound. These results show a good agreement with the DIS experimental data throughout the low values of $x$. Results can be applied to the LHeC region for analyses of ultra-high energy processes.
\end{abstract}

\section{Introduction}

The reduced cross section is defined into the transverse and longitudinal structure functions, $F_{2}\left(x, Q^{2}\right)$ and $F_{L}\left(x, Q^{2}\right)$, by the following form

$$
\widetilde{\sigma}\left(x, Q^{2}\right)=F_{2}\left(x, Q^{2}\right)-\frac{y^{2}}{Y_{+}} F_{L}\left(x, Q^{2}\right),
$$

where $Y_{+}=1+(1-y)^{2}, y=Q^{2} / x s$ denotes the inelasticity and $s$ stands for the center-of-mass squared energy of incoming electrons and protons. As usual $x$ is the Bjorken scaling parameter and $Q^{2}$ is the four momentum transfer in a deep inelastic scattering process. For fixed $Q^{2}$, the reduced cross section rises with decreasing $x$. However, at high- $y$ (very low $x$ ) a characteristic bending of the reduced cross section is observed, which it is attributed to the contribution due to the longitudinal structure function.

The longitudinal structure function $F_{L}$ is proportional to the cross section for the interaction of the longitudinally polarized virtual photon with a proton. This observable is interest since it is directly sensitive to the gluon density. The longitudinal structure function $F_{L}\left(x, Q^{2}\right)$ obtained as a consequence of the violation of Callan- Gross relation [1] and is defined as $F_{L}\left(x, Q^{2}\right)=F_{2}\left(x, Q^{2}\right)-2 x F_{1}\left(x, Q^{2}\right)$. Beyond the parton model the $F_{L}$ effects can be sizable, hence it can not be longer neglected. Also, the longitudinal structure function is predominant in cosmic neutrinohadron cross section scattering [2]. This behavior for the longitudinal structure function will be checked in high energy process such as the Large Hadron electron Collider (LHeC) project which runs to beyond a $\mathrm{TeV}$ in centerof-mass energy [3].

Data on $F_{L}$ are generally difficult to extract from reduced cross section measurements. This procedure require high-precision cross section measurements at the

\footnotetext{
*brezaei@razi.ac.ir

${ }^{\dagger}$ Electronic address: grboroun@gmail.com; boroun@razi.ac.ir
}

same values of $x$ and $Q^{2}$ but at different center-of-mass energy of the incoming beams [4]. Recently, the new data on the proton longitudinal structure function has been taken from $\mathrm{H} 1$ experiment at HERA. HERA collected ep collision data with the $\mathrm{H} 1$ detector at a electron beam energy of $27.6 \mathrm{GeV}$ and proton beam energies of 920,575 and $460 \mathrm{GeV}$, which allowed a measurement of structure functions at $x$ values $6.5 \times 10^{-4} \leq x \leq 0.65$ and $Q^{2}$ values $35 \mathrm{GeV}^{2} \leq Q^{2} \leq 800 \mathrm{GeV}^{2}[5]$.

It is well known that the corresponding Wilson coefficients for the longitudinal structure functions were calculated in LO, NLO and next-to-next-to-leading order (NNLO) [6-8]. Inclusion of higher order kernels in the study of the longitudinal structure function is the particular important for studying the physical processes at new colliders [3]. Authors in Refs.[9-14] presented a set of formula to extract the longitudinal structure function $F_{L}\left(x, Q^{2}\right)$ from the transverse structure function $F_{2}$ and its derivative $d F_{2} / d \ln Q^{2}$ at low- $x$.

Recently, several methods have been proposed that the longitudinal structure function $F_{L}$ can be related to the nucleon structure function $F_{2}$ and the $F_{2}$ scaling violation at low values of $x$ [15-17]. These approaches solve the evolution equations for Laplace and Mellin transform of the parton densities and subsequently invert the transforms back to $x$-space, respectively. In Ref.[15], an analytical relation has been derived for calculating the longitudinal structure function within the Laplace-transform method at low $x$ values. Moreover, this method may also use to extract nonliner corrections to the longitudinal structure function from new collider data. In Refs.[16-17], authors report relations between the longitudinal structure function and the proton structure function based on the Mellin-transform method at leading order (LO) and next -to- leading order (NLO) analysis respectively. The obtained results are based on an analytical solution for the proton structure function in Refs.[18-19]. They have been suggested a new parameterization of the proton structure function at asymptotic low $x$ values. The obtained results indicate a good agreement with the deep inelastic scattering (DIS) data on the 
reduced cross section.

In next section we describe the basic theory to extract the longitudinal structure function from the AltarelliMartinelli equation [20] and the distribution functions by solving the DGLAP evolution equations. Then, the analytical solution of the master equation for the longitudinal structure function into the gluonic and singlet terms at LO up to NNLO are devoted respectively. Finally, an analytical analysis of our solutions is presented and the obtained results are compared with the experimental data which are followed by results and discussion. In the present paper the behavior of the longitudinal structure function in $x$-space directly is investigated at LO up to NNLO analysis. We extend the method using the Regge technique. The Regge behavior of the singlet and gluon distributions is introduced by using the power law behavior as $F_{2}^{s} \sim x^{-\lambda_{s}}$ and $G \sim x^{-\lambda_{g}}$. We note that the behavior of distributions with a $Q^{2}$ independent value for exponents obeys the DGLAP equations [21] when $x^{-\lambda_{s, g}} \gg 1$. This behavior at low- $x$ is well explained in terms of Regge-like ansatz [22-23]. In this region, the Regge behavior of the singlet and gluon distribution is controlled by pomeron exchange. Let us take the power law behavior for distribution functions as $F_{2}^{s}\left(x, Q^{2}\right)=A_{s}\left(Q^{2}\right) x^{-\lambda_{s}}$ and $G\left(x, Q^{2}\right)=A_{g}\left(Q^{2}\right) x^{-\lambda_{g}}$, that exponents $\lambda_{s}$ and $\lambda_{g}$ are given as the following derivatives:

$$
\lambda_{s}=\frac{\partial \ln F_{2}^{s}\left(x, Q^{2}\right)}{\partial \ln (1 / x)}, \lambda_{g}=\frac{\partial \ln G\left(x, Q^{2}\right)}{\partial \ln (1 / x)} .
$$

\section{Theory}

The longitudinal structure function projected from the hadronic tensor can be considered with combination of the metric and the spacelike momentum transferred by the virtual photon $\left(g_{\mu \nu}-q_{\mu} q_{\nu} / q^{2}\right)$. It is proportional to hadronic tensor as follows

$$
F_{L}\left(x, Q^{2}\right) / x=\frac{8 x^{2}}{Q^{2}} p_{\mu} p_{\nu} W_{\mu \nu}\left(x, Q^{2}\right) .
$$

Here $p^{\mu}\left(p^{\nu}\right)$ is the hadron momentum and $W^{\mu \nu}$ is the hadronic tensor. The total cross section of a hadronic process can be written as the sum of the contributions of each parton type (quarks, antiquarks, and gluons) carrying a fraction of the hadronic total momentum. In DIS it reads

$$
d \sigma_{H}(p)=\sum_{i} \int d y d \hat{\sigma}_{i}(y p) \Pi_{i}^{0}(y)
$$

where $d \hat{\sigma}_{i}$ is the cross section corresponding to the parton $i$ and $\Pi_{i}^{0}(y)$ is the probability of finding this parton in the hadron target with the momentum fraction $y$. Now, taking into account the kinematical constrains one gets the relation between the hadronic and the partonic structure functions

$$
\begin{aligned}
f_{j}\left(x, Q^{2}\right)= & \sum_{i} \int_{x}^{1} \frac{d y}{y} \mathrm{f}_{j}\left(\frac{x}{y}, Q^{2}\right) \Pi_{i}^{0}(y) \\
& =\sum_{i} \mathrm{f}_{j} \otimes \Pi_{i}^{0}(y) \quad, j=2, L,
\end{aligned}
$$

where $\mathrm{f}_{j}\left(x, Q^{2}\right)=F_{j}\left(x, Q^{2}\right) / x$ and the symbol $\otimes$ denotes a convolution according to the usual prescription, $f(x) \otimes g(x)=\int_{x}^{1} \frac{d y}{y} f(y) g\left(\frac{x}{y}\right)$. Equation (5) expresses the hadronic structure functions as the convolution of the partonic structure function which are calculable in perturbation theory.

In perturbative QCD, the longitudinal structure function in terms of the coefficient functions is given by [20]

$$
x^{-1} F_{L}=C_{L, n s} \otimes q_{n s}+<e^{2}>\left(C_{L, q} \otimes q_{s}+C_{L, g} \otimes g\right),
$$

where the non-singlet quark distribution, $x q_{n s}$, become negligibly small in comparison with the singlet and gluon distribution functions, $x q_{s}$ and $x g$, at low values of $x$ and can be ignored. $\left\langle e^{k}\right\rangle$ is the average of the charge $e^{k}$ for the active quark flavors, $<e^{k}>=n_{f}^{-1} \sum_{i=1}^{n_{f}} e_{i}^{k}$. The perturbative expansion of the coefficient functions can be written as [7]

$$
C_{L, a}\left(\alpha_{s}, x\right)=\sum_{n=1} a(t)^{n} c_{L, a}^{n}(x),
$$

where $n$ is the order in the running coupling constant. The running coupling constant in the high-loop corrections of the above equation is expressed entirely thorough the variable $a(t)$, as $a(t)=\frac{\alpha_{s}}{4 \pi}$. The explicit expression for the coefficient functions in LO up to NNLO are relegated in Appendix A.

The running coupling constant $\alpha_{s}$ has the following forms in NLO up to NNLO respectively [24]

$$
\alpha_{s}^{\mathrm{NLO}}=\frac{4 \pi}{\beta_{0} t}\left[1-\frac{\beta_{1} \ln t}{\beta_{0}^{2} t}\right]
$$

and

$$
\begin{aligned}
\alpha_{s}^{\mathrm{NNLO}=} & \frac{4 \pi}{\beta_{0} t}\left[1-\frac{\beta_{1} \ln t}{\beta_{0}^{2} t}+\frac{1}{\left(\beta_{0} t\right)^{2}}\left[\left(\frac{\beta_{1}}{\beta_{0}}\right)^{2}\right.\right. \\
& \left.\left.\left(\ln ^{2} t-\ln t+1\right)+\frac{\beta_{2}}{\beta_{0}}\right]\right] .
\end{aligned}
$$

where $\beta_{0}=\frac{1}{3}\left(33-2 n_{f}\right), \beta_{1}=102-\frac{38}{3} n_{f}$ and $\beta_{2}=\frac{2857}{6}-\frac{6673}{18} n_{f}+\frac{325}{54} n_{f}^{2}$ are one-loop, two-loop and three-loop corrections to the QCD $\beta$-function and $n_{f}$ denotes the number of effectively massless flavours. The variable $t$ is defined as $t=\ln \left(\frac{Q^{2}}{\Lambda^{2}}\right)$. We take the $n_{f}=4$ for $m_{c}^{2}<\mu^{2}<m_{b}^{2}$ and adjust the QCD cutoff parameter $\Lambda$ at each heavy quark mass threshold, $\mu^{2}=m_{c}^{2}$. This parameter has been extracted from the 
strong coupling constat at the $\mathrm{Z}$ boson mass scale for the NLO and NNLO approximation in accordance with the table III.

The Dokshitzer-Gribov-Lipatov-Altarelli-Parisi (DGLAP) [21] evolution equations are fundamental tools to study the $Q^{2}$ - and $x$-evolutions of structure functions. The structure function reflects the momentum distributions of the partons in the nucleon. It is also important to know the gluon distribution inside a hadron at low- $x$ because gluons are expected to be dominant in this region. Some analytical solutions of the polarized and unpolarized DGLAP evolution equations have been reported in last years [25-26] with considerable phenomenological success. The flavor-singlet quark density of a hadron is given by

$$
q_{s}=\sum_{i=1}^{n_{f}}\left[q_{i}+\bar{q}_{i}\right]
$$

where $q_{i}$ and $\bar{q}_{i}$ represent the number distribution of quarks and antiquarks, respectively, in the fractional hadron momentum $x$. The subscripts $i$ indicate the flavor of the quarks and antiquarks. In the common $\overline{\mathrm{MS}}$ renormalization scheme the proton structure function, extracted from the DIS ep process, can be written as the sum of a flavour singlet. Using the fact that the nonsinglet contribution $F_{2}^{N S}$ can be ignored safely at low values of $x$ so we will have,

$$
\begin{aligned}
\frac{F_{2}\left(x, Q^{2}\right)}{x} \simeq & \frac{1}{x}\left(F_{2, s}\left(x, Q^{2}\right)+F_{2, g}\left(x, Q^{2}\right)\right) \\
& =<e^{2}>\left(C_{2, s}\left(x, Q^{2}\right) \otimes q_{s}\left(x, Q^{2}\right)\right. \\
& \left.+C_{2, g}\left(x, Q^{2}\right) \otimes g\left(x, Q^{2}\right)\right)
\end{aligned}
$$

where the corresponding gluon density is denoted by $g\left(x, Q^{2}\right)$ and $C_{2, i}(i=s, g)$ are the common Wilson coefficient functions. In the space-like region, the proton structure function can be expressed in terms of quark and gluon densities

$$
\begin{aligned}
F_{2}\left(x, Q^{2}\right)= & x \sum_{j=1}^{n_{f}} e_{j}^{2} \int_{x}^{1} \frac{d z}{z}\left[\frac{1}{n_{f}} q_{s}\left(\frac{x}{z}, \mu_{f}^{2}\right) C_{2, s}\left(z, \frac{Q^{2}}{\mu_{f}^{2}}\right)\right. \\
& \left.+g\left(\frac{x}{z}, \mu_{f}^{2}\right) C_{2, g}\left(z, \frac{Q^{2}}{\mu_{f}^{2}}\right)\right] .
\end{aligned}
$$

The scale $\mu_{f}$ denotes the factorization scale. Considering coupling constant renormalization, the proton structure function is expressed as follows [27]

$$
\begin{gathered}
F_{2}\left(x, Q^{2}\right)=\left[C_{2, s}\left(\alpha_{s}\left(\mu_{r}^{2}\right), \frac{Q^{2}}{\mu_{f}^{2}}, \frac{\mu_{f}^{2}}{\mu_{r}^{2}}\right) \otimes q_{s}\left(\alpha_{s}\left(\mu_{r}^{2}\right), \frac{\mu_{f}^{2}}{\mu^{2}}, \frac{\mu_{f}^{2}}{\mu_{r}^{2}}\right)\right. \\
\left.+C_{2, g}\left(\alpha_{s}\left(\mu_{r}^{2}\right), \frac{Q^{2}}{\mu_{f}^{2}}, \frac{\mu_{f}^{2}}{\mu_{r}^{2}}\right) \otimes g\left(\alpha_{s}\left(\mu_{r}^{2}\right), \frac{\mu_{f}^{2}}{\mu^{2}}, \frac{\mu_{f}^{2}}{\mu_{r}^{2}}\right)\right](x),
\end{gathered}
$$

which the renormalized parton density is defined by the following form

$$
\begin{aligned}
& f_{l}\left(z, \alpha_{s}\left(\mu_{r}^{2}\right), \frac{\mu_{f}^{2}}{\mu^{2}}, \frac{\mu_{f}^{2}}{\mu_{r}^{2}}\right)=\sum_{k=q, g}\left(\Gamma_{l k}\left(\alpha_{s}\left(\mu_{r}^{2}\right), \frac{\mu_{f}^{2}}{\mu^{2}}, \frac{\mu_{f}^{2}}{\mu_{r}^{2}}, \epsilon\right) \otimes \widehat{f}_{k}\right)(z), \\
& f_{q}=q_{s} \text { and } f_{g}=g \text { for } l=q, g .
\end{aligned}
$$

Here $\mu_{r}$ is the renormalization scale, $\epsilon=n-4$ represents the collinear singularities and $\widehat{f}_{k}$ is defined as the bare parton density. Due to changes in the scales $\mu_{r}$ and $\mu_{f}$, the changes in the parton densities and the coefficient functions can be expressed in terms of the renormalization group equation [28]. The renormalization group equation of the parton densities and the scale dependence of the coefficient functions causes the parton distribution functions to be expressed in terms of the splitting functions $P_{i j}\left(x, \alpha_{s}\right)$. The evolution equations of distribution functions in the singlet sector are given by

$$
Q^{2} \frac{\partial}{\partial Q^{2}}\left(\begin{array}{c}
F_{2}^{s}\left(x, Q^{2}\right) \\
G\left(x, Q^{2}\right)
\end{array}\right)=\left(\begin{array}{cc}
P_{q q}\left(x, \alpha_{s}\right) & P_{q g}\left(x, \alpha_{s}\right) \\
P_{g q}\left(x, \alpha_{s}\right) & P_{g g}\left(x, \alpha_{s}\right)
\end{array}\right) \otimes\left(\begin{array}{c}
F_{2}^{s}\left(x, Q^{2}\right) \\
G\left(x, Q^{2}\right)
\end{array}\right)
$$

where $G\left(x, Q^{2}\right)$ is the gluon distribution function defined into the gluon density by $G\left(x, Q^{2}\right)=x g\left(x, Q^{2}\right)$. For the high-order contribution to scaling violation of distribu- tion functions, we extend the discussion to the NNLO level. So that the high-order correction to the coupled DGLAP evolution equations can be written as 


$$
\begin{aligned}
Q^{2} \frac{\partial}{\partial Q^{2}} F_{2}^{s}\left(x, Q^{2}\right)= & {\left[\frac{\alpha_{s}\left(Q^{2}\right)}{4 \pi} P_{q q}^{0}(x)+\left(\frac{\alpha_{s}\left(Q^{2}\right)}{4 \pi}\right)^{2} P_{q q}^{1}(x)+\left(\frac{\alpha_{s}\left(Q^{2}\right)}{4 \pi}\right)^{3} P_{q q}^{2}(x)\right] \otimes F_{2}^{s}\left(x, Q^{2}\right) } \\
& +\left[\frac{\alpha_{s}\left(Q^{2}\right)}{4 \pi} P_{q g}^{0}(x)+\left(\frac{\alpha_{s}\left(Q^{2}\right)}{4 \pi}\right)^{2} P_{q g}^{1}(x)+\left(\frac{\alpha_{s}\left(Q^{2}\right)}{4 \pi}\right)^{3} P_{q g}^{2}(x)\right] \otimes G\left(x, Q^{2}\right) . \\
Q^{2} \frac{\partial}{\partial Q^{2}} G\left(x, Q^{2}\right)= & {\left[\frac{\alpha_{s}\left(Q^{2}\right)}{4 \pi} P_{g q}^{0}(x)+\left(\frac{\alpha_{s}\left(Q^{2}\right)}{4 \pi}\right)^{2} P_{g q}^{1}(x)+\left(\frac{\alpha_{s}\left(Q^{2}\right)}{4 \pi}\right)^{3} P_{g q}^{2}(x)\right] \otimes F_{2}^{s}\left(x, Q^{2}\right) } \\
& +\left[\frac{\alpha_{s}\left(Q^{2}\right)}{4 \pi} P_{g g}^{0}(x)+\left(\frac{\alpha_{s}\left(Q^{2}\right)}{4 \pi}\right)^{2} P_{g g}^{1}(x)+\left(\frac{\alpha_{s}\left(Q^{2}\right)}{4 \pi}\right)^{3} P_{g g}^{2}(x)\right] \otimes G\left(x, Q^{2}\right) .
\end{aligned}
$$

The splitting functions $P_{i j}^{n}(n=0,1,2)$ are the AltarelliParisi splitting kernels at one, two and three loops corrections. The explicit forms of the splitting functions at LO up to NNLO analysis are given in Appendix B. For brevity the leading-order contribution to the coupled DGLAP evolution equation can be written

$$
\begin{aligned}
\frac{4 \pi}{\alpha_{s}\left(Q^{2}\right)} \frac{\partial G\left(x, Q^{2}\right)}{\partial \ln Q^{2}}= & \frac{33-2 n_{f}}{3} G\left(x, Q^{2}\right)+12 G\left(x, Q^{2}\right) \ln \frac{1-x}{x}+12 x \int_{x}^{1} \frac{d z}{z-x}\left(\frac{G\left(z, Q^{2}\right)}{z}-\frac{G\left(x, Q^{2}\right)}{x}\right) \\
& +12 x \int_{x}^{1} G\left(z, Q^{2}\right)\left(\frac{z}{x}-2+\frac{x}{z}-\frac{x^{2}}{z^{2}}\right) \frac{d z}{z^{2}}+\frac{8}{3} \int_{x}^{1} F_{2}^{s}\left(z, Q^{2}\right)\left(1+\left(1-\frac{x}{z}\right)^{2}\right) \frac{d z}{z} \\
\frac{4 \pi}{\alpha_{s}\left(Q^{2}\right)} \frac{\partial F_{2}^{s}\left(x, Q^{2}\right)}{\partial \ln Q^{2}}= & 4 F_{2}^{s}\left(x, Q^{2}\right)+\frac{16}{3} F_{2}^{s}\left(x, Q^{2}\right) \ln \frac{1-x}{x}+\frac{16}{3} x \int_{x}^{1} \frac{d z}{z-x}\left(\frac{F_{2}^{s}\left(z, Q^{2}\right)}{z}-\frac{F_{2}^{s}\left(x, Q^{2}\right)}{x}\right) \\
& -\frac{8}{3} x \int_{x}^{1} F_{2}^{s}\left(z, Q^{2}\right)\left(1+\frac{x}{z}\right) \frac{d z}{z^{2}}+2 n_{f} x \int_{x}^{1} G\left(z, Q^{2}\right)\left(1-2 \frac{x}{z}+2 \frac{x^{2}}{z^{2}}\right) \frac{d z}{z} .
\end{aligned}
$$

Now we consider the behavior of the longitudinal structure function with respect to the hard-pomeron behavior [22-23,31] by using the parametrization of $F_{2}\left(x, Q^{2}\right)$ and $G\left(x, Q^{2}\right)$ presented in Refs.[19] and [32] respectively. This study is based on two steps:

1) In the first step of the analysis, we only present the longitudinal structure function behavior by using the gluon distribution function and its logarithmic derivative [32].

2) Then the longitudinal structure function have been extracted at low $x$ from the proton structure function $F_{2}\left(x, Q^{2}\right)$ and the logarithmic derivative $d F_{2}\left(x, Q^{2}\right) / d \ln \left(Q^{2}\right)[19]$.

The next-to-leading order corrections are the standard approximations for the most important processes. The corresponding one- and two-loop splitting functions have been known for a long time. As in Refs. [7-8, 29-30,33], the authors have been reported the complete two- and three-order coefficient functions for the longitudinal structure functions in deep inelastic scattering (DIS). The NNLO corrections need to be included, however, in order to arrive to quantitatively reliable predictions for ultra low values of $x$ at present and future high-energy colliders. These corrections are known not only for the distribution functions but also for the longitudinal coefficient functions in deep-inelastic scattering (DIS). Now, a detailed analysis has been performed to find analytical solutions of the longitudinal structure function into the distributions and those derivatives, using the hard-pomeron behavior, at LO up to NNLO approximations.

\section{Method}

The following parameterization of the deep inelastic scattering structure function $F_{2}\left(x, Q^{2}\right)$ defined by

$$
F_{2}\left(x, Q^{2}\right) \sim \sum_{i} A_{i}\left(Q^{2}\right) x^{-\lambda_{i}}
$$

The singlet part of the structure function is controlled by pomeron exchange. Here the $i=0$ term is hardpomeron and $i=1$ is soft-pomeron exchange [21-22]. The effective intercept behavior, at low values of $x$, exhibiting for the fast growth of the singlet structure function. The exponent $\lambda_{s}$ is found to be $\simeq 0.33$ in Refs. [34-35]. It can 
be recasted into the symbolic form as

$$
F_{2}\left(x, Q^{2}\right) \propto x^{-\lambda_{s}} .
$$

The low- $x$ behavior of the gluon distribution function also is dominated with hard-pomeron intercept as

$$
G\left(x, Q^{2}\right) \propto x^{-\lambda_{g}}
$$

where $\lambda_{g} \simeq 0.43[22-23,34-35]$. Based on the hardpomeron behavior for the distribution functions, let us put Eqs.(17) and (18) in r.h.s of Eqs.(14). After doing the integration over $z$, These equations can be rewritten in a convolution form as

$$
\begin{aligned}
\frac{\partial G\left(x, Q^{2}\right)}{\partial \ln Q^{2}}= & G\left(x, Q^{2}\right) \Phi_{g g}\left(x, Q^{2}\right) \\
& +F_{2}\left(x, Q^{2}\right) \Theta_{g q}\left(x, Q^{2}\right)
\end{aligned}
$$

and

$$
\begin{aligned}
\frac{\partial F_{2}\left(x, Q^{2}\right)}{\partial \ln Q^{2}}= & F_{2}\left(x, Q^{2}\right) \Phi_{q q}\left(x, Q^{2}\right) \\
& +G\left(x, Q^{2}\right) \Theta_{q g}\left(x, Q^{2}\right) .
\end{aligned}
$$

On the other hand, the Altarelli- Martinelli equation for the longitudinal stricture function at low $x$ values is given by the similar method as

$$
\begin{aligned}
F_{L}\left(x, Q^{2}\right)= & F_{2}\left(x, Q^{2}\right) I_{L, q}\left(x, Q^{2}\right) \\
& +G\left(x, Q^{2}\right) I_{L, g}\left(x, Q^{2}\right) .
\end{aligned}
$$

The analytical results for the compact form of the kernels $(\Phi, \Theta$ and $I)$ at LO up to NNLO are given in Appendix C.

\section{Gluonic Formalism}

Using the above kernels and Eqs.(19) and (21), we can calculate the $F_{L}$ structure function into the gluon distribution and its derivative [36-37]. For this purpose the ratio $\frac{F_{2}\left(x, Q^{2}\right)}{G\left(x, Q^{2}\right)}$ is determined by solving the DGLAP evolution equation for the gluon distribution function (i.e.,
Eq.(19)) and the Altarelli- Martinelli equation for the longitudinal stricture function (i.e. Eq.(21)). Therefore the $F_{L}$ using the gluonic terms extracted by the following form

$$
\begin{aligned}
F_{L}\left(x, Q^{2}\right)= & \frac{I_{L, q}\left(x, Q^{2}\right)}{\Theta_{g q}\left(x, Q^{2}\right)} \frac{\partial G\left(x, Q^{2}\right)}{\partial \ln Q^{2}}+\left\{I_{L, g}\left(x, Q^{2}\right)\right. \\
& \left.-\Phi_{g g}\left(x, Q^{2}\right) \frac{I_{L, q}\left(x, Q^{2}\right)}{\Theta_{g q}\left(x, Q^{2}\right)}\right\} G\left(x, Q^{2}\right) .
\end{aligned}
$$

The accuracy this relation can be checked for certain parametrization of the gluon distribution function and its derivatives.

In the following, we will present our analytical method based on the newly-proton structure function which strongly violate the Froissart boundary [38]. We used the hard-pomeron behavior for the input singlet and gluon exponents to determine the parton distribution behavior at any $Q^{2}$ values. Having the exponents and using the proton structure function (i.e., Appendix D), one can extract the longitudinal structure function as a function of $x$ at any desired $Q^{2}$ value.

\section{Singlet Formalism}

One can rewrite Eqs.(20) and (21) with respect to the proton structure function $F_{2}\left(x, Q^{2}\right)$ and its derivative $\partial F_{2}\left(x, Q^{2}\right) / \partial \ln Q^{2}$. Then we will have

$$
\begin{aligned}
F_{L}\left(x, Q^{2}\right)= & \frac{I_{L, g}\left(x, Q^{2}\right)}{\Theta_{q g}\left(x, Q^{2}\right)} \frac{\partial F_{2}\left(x, Q^{2}\right)}{\partial \ln Q^{2}}+\left\{I_{L, q}\left(x, Q^{2}\right)\right. \\
& \left.-\Phi_{q q}\left(x, Q^{2}\right) \frac{I_{L, g}\left(x, Q^{2}\right)}{\Theta_{q g}\left(x, Q^{2}\right)}\right\} F_{2}\left(x, Q^{2}\right)
\end{aligned}
$$

Eqs.(28-40) (in Appendixes A-C) help to estimate the longitudinal proton structure function in the leading order up to the next-to-next-to-leading order approximation, as we get 


$$
\begin{aligned}
F_{L}^{L O}\left(x, Q^{2}\right)= & \frac{1}{2} \frac{c_{L, g}^{L O}(x) \odot x^{\lambda_{g}}}{P_{q g}^{L O}(x) \odot x^{\lambda_{g}}} \frac{\partial F_{2}\left(x, Q^{2}\right)}{\partial \ln Q^{2}}+\left\{\frac{\alpha_{s}}{4 \pi} c_{L, q}^{L O}(x) \odot x^{\lambda_{s}}-\left[\frac{\alpha_{s}}{2 \pi} P_{q q}^{L O}(x) \odot x^{\lambda_{s}}\right] \frac{1}{2} \frac{c_{L, g}^{L O}(x) \odot x^{\lambda_{g}}}{P_{q g}^{L O}(x) \odot x^{\lambda_{g}}}\right\} F_{2}\left(x, Q^{2}\right), \\
F_{L}^{N L O}\left(x, Q^{2}\right)= & \frac{1}{2} \frac{\left[c_{L, g}^{L O}(x)+\frac{\alpha_{s}}{4 \pi} c_{L, g}^{N O}(x)\right] \odot x^{\lambda_{g}}}{\left[P_{q g}^{L O}(x)+\frac{\alpha_{s}}{2 \pi} P_{q g}^{N L O}(x)\right] \odot x^{\lambda_{g}}} \frac{\partial F_{2}\left(x, Q^{2}\right)}{\partial \ln Q^{2}}+\left\{\frac{\alpha_{s}}{4 \pi}\left[c_{L, q}^{L O}(x)+\frac{\alpha_{s}}{4 \pi} c_{L, q}^{N L O}(x)\right] \odot x^{\lambda_{s}}\right. \\
& \left.-\left[\frac{\alpha_{s}}{2 \pi}\left(P_{q q}^{L O}(x)+\frac{\alpha_{s}}{2 \pi} P_{q q}^{N L O}(x)\right) \odot x^{\lambda_{s}}\right] \frac{1}{2} \frac{\left[c_{L, g}^{L O}(x)+\frac{\alpha_{s}}{4 \pi} c_{L, g}^{N O}(x)\right] \odot x^{\lambda_{g}}}{\left[P_{q g}^{L O}(x)+\frac{\alpha_{s}}{2 \pi} P_{q g}^{N L O}(x)\right] \odot x^{\lambda_{g}}}\right\} F_{2}\left(x, Q^{2}\right),
\end{aligned}
$$

and

$$
\begin{aligned}
F_{L}^{N N L O}\left(x, Q^{2}\right)= & \frac{1}{2} \frac{\left[c_{L, g}^{L O}(x)+\frac{\alpha_{s}}{4 \pi} c_{L, g}^{N L O}(x)+\left(\frac{\alpha_{s}}{4 \pi}\right)^{2} c_{L, g}^{N N O}(x)\right] \odot x^{\lambda_{g}}}{\left[P_{q g}^{L O}(x)+\frac{\alpha_{s}}{2 \pi} P_{q g}^{N L O}(x)+\left(\frac{\alpha_{s}}{2 \pi}\right)^{2} P_{q g}^{N N L O}(x)\right] \odot x^{\lambda_{g}}} \frac{\partial F_{2}\left(x, Q^{2}\right)}{\partial \ln Q^{2}} \\
& +\left\{\frac{\alpha_{s}}{4 \pi}\left[c_{L, q}^{L O}(x)+\frac{\alpha_{s}}{4 \pi} c_{L, q}^{N L O}(x)+\left(\frac{\alpha_{s}}{4 \pi}\right)^{2} c_{L, q}^{N N L O}(x)\right] \odot x^{\lambda_{s}}\right. \\
& -\left[\frac{\alpha_{s}}{2 \pi}\left(P_{q q}^{L O}(x)+\frac{\alpha_{s}}{2 \pi} P_{q q}^{N L O}(x)+\left(\frac{\alpha_{s}}{2 \pi}\right)^{2} P_{q q}^{N N L O}(x)\right) \odot x^{\lambda_{s}}\right] \\
& \frac{1}{2} \frac{\left[c_{L, g}^{L O}(x)+\frac{\alpha_{s}}{4 \pi} c_{L, g}^{N L O}(x)+\left(\frac{\alpha_{s}}{4 \pi}\right)^{2} c_{L, g}^{N N O}(x)\right] \odot x^{\lambda_{g}}}{\left.\left[P_{q g}^{L O}(x)+\frac{\alpha_{s}}{2 \pi} P_{q g}^{N L O}(x)+\left(\frac{\alpha_{s}}{2 \pi}\right)^{2} P_{q g}^{N N L O}(x)\right] \odot x^{\lambda_{g}}\right\} F_{2}\left(x, Q^{2}\right),}
\end{aligned}
$$

where we describe the following statement in the convenient form for further discussion $f(x) \odot g(x) \equiv \int_{x}^{1}(d y / y) f(y) g(y)$.

By using the leading-order splitting and coefficient functions presented in Appendixes A,B and $\mathrm{C}$, the longitudinal structure function is given by the following form

$$
\begin{aligned}
F_{L}^{L O}\left(x, Q^{2}\right)= & \frac{12}{5} \frac{\int_{x}^{1} z^{\lambda_{g}+1} d z}{\int_{x}^{1}\left(z^{2}+(1-z)^{2}\right) z^{\lambda_{g}} d z} \frac{\partial F_{2}\left(x, Q^{2}\right)}{\partial \ln Q^{2}}\left[\equiv \text { Derivative Eq.(42) into } \ln Q^{2}\right] \\
& +\left\{\frac{\alpha_{s}}{4 \pi} \int_{x}^{1} 8 n_{f}(1-z) z^{\lambda_{s}+1} d z-\left[\frac { \alpha _ { s } } { 4 \pi } \left\{4+\frac{16}{3} \ln \left(\frac{1-x}{x}\right)+\frac{16}{3} \int_{x}^{1} \frac{z^{\lambda_{s}}-z^{-1}}{1-z} d z\right.\right.\right. \\
& \left.\left.\left.-\frac{8}{3} \int_{x}^{1}(1+z) z^{\lambda_{s}} d z\right\}\right] \frac{12}{5} \frac{\int_{x}^{1} z^{\lambda_{g}+1} d z}{\int_{x}^{1}\left(z^{2}+(1-z)^{2}\right) z^{\lambda_{g}} d z}\right\} F_{2}\left(x, Q^{2}\right)[\equiv \text { Eq. (42)]. }
\end{aligned}
$$

The NLO and NNLO longitudinal structure functions are too lengthy to include here and we present them in compact forms of Eqs.(24). Consequently, one can obtain the longitudinal structure function from the parameterization of $F_{2}$ (i.e., Eq.(42)) at LO, NLO and NNLO approximations respectively.

\section{Results and Discussion}

In this section, we shall present our results that have been obtained for the longitudinal structure function $F_{L}\left(x, Q^{2}\right)$ using the hard-pomeron behavior of the distribution functions to find an analytical solution for the combined DGLAP and Altarelli- Martinelli equations. The proton structure function and its derivative are supposed to be known with respect to the parameterization represented in Ref.[19]. This parameterization obtained from a combined fit of the H1 and ZEUS collaborations data [41] in a range of the kinematical variables $x<0.01$ and $0.15 \mathrm{GeV}^{2}<Q^{2}<3000 \mathrm{GeV}^{2}$.

We use the values of $\lambda_{s} \simeq 0.33$ and $\lambda_{g} \simeq 0.43$ within the range of $Q^{2}$ under study [34-35, 42-43]. The coupling constant defined via the $n_{f}=4$ definition of $\Lambda_{Q C D}$ for the ZEUS data [41] and the MRST set of partons [44]. The values of $\Lambda_{Q C D}$ at LO up to NNLO are displayed in Table III respectively. The predictions for the longitudinal structure function, in the kinematic range where it has been measured by $H 1$ collaboration [5], are com- 
puted and compared at low values of $x$.

The results at LO up to NNLO are presented in Fig.1 and compared with the $H 1$ data [5]. In comparison with Refs.[16-17], the results have been depicted at fixed value of the invariant mass $\mathrm{W}$ (i.e. $W=230 \mathrm{GeV}$ ). As can be seen in this figure, the results are comparable with the $\mathrm{H} 1$ data in the interval $1 \mathrm{GeV}^{2}<Q^{2}<500 \mathrm{GeV}^{2}$ in both NLO and NNLO analysis. At all $Q^{2}$ values the NNLO extracted longitudinal structure functions are in a good agreement with experimental data. The $x$-evolution results of $F_{L}$ structure function are depicted in Fig.2 where we have compared these results at LO, NLO and NNLO with $\mathrm{H} 1$ data. As can be seen in all figures, the increase of these calculations for the longitudinal structure function $F_{L}\left(x, Q^{2}\right)$ towards low $x$ are consistent with theoretical investigations of ultra-high energy processes with cosmic neutrinos. Also the NNLO results are in the context of the Froissart bound at very low $x$ values.

In what follows the ratio $F_{L}\left(x, Q^{2}\right) / F_{2}\left(x, Q^{2}\right)$ is calculated and presented in Figs. 3 and 4. Here we use directly the parametrization of $F_{2}$ from Ref.[19] in the ratio $\frac{F_{L}}{F_{2}}$. In these figures the ratio of the structure functions are compared with the H1 data [5]. The error bars of the ratio $\frac{F_{L}}{F_{2}}$ are determined by the following form [45]

$$
\Delta\left(\frac{F_{L}}{F_{2}}\right)=\frac{F_{L}}{F_{2}} \sqrt{\left(\frac{\Delta F_{L}}{F_{L}}\right)^{2}+\left(\frac{\Delta F_{2}}{F_{2}}\right)^{2}},
$$

where $\Delta F_{L}$ and $\Delta F_{2}$ are collected from the $\mathrm{H} 1$ experimental data in Ref.[5]. The NNLO result obtained of the ratio $\frac{F_{L}}{F_{2}}$ is comparable with the color dipole model bound [46]. The good agreement between this method at NNLO analysis and the experimental data indicates that these results have a bound asymptotic behavior and it is compatible with the color dipole model bound ( $\left.\frac{F_{L}\left(x, Q^{2}\right)}{F_{2}\left(x, Q^{2}\right)}<0.27\right)$. In Fig. 4 we observe that the ratio at NNLO is almost independent of $x$-evolution. This strict bound is comparable with the color dipole bound and experimental data in $x-Q^{2}$ domain.

The measurements of longitudinal structure function $F_{L}$ are used to determine the DIS cross section ratio $R\left(x, Q^{2}\right)$. The value of $R$ depends on the ratio of the longitudinal to transversal cross sections, as

$$
\begin{aligned}
R\left(x, Q^{2}\right)= & \frac{\sigma_{L}\left(x, Q^{2}\right)}{\sigma_{T}\left(x, Q^{2}\right)} \\
& =\frac{F_{L}\left(x, Q^{2}\right)}{F_{2}\left(x, Q^{2}\right)-F_{L}\left(x, Q^{2}\right)},
\end{aligned}
$$

which $\sigma_{L}$ and $\sigma_{T}$ are the absorption cross section of longitudinally and transversely polarized virtual photon by proton. This ratio is expected to vanish at large $Q^{2}$ and moderate $x$ in the naive parton model, but is nonzero at low values of $x$. It dues to the fact that partons can carry transverse momentum [47]. In Figs.(5) and (6) we have also studied the behavior of the ratio $R$ at low $x$ in LO, NLO and NNLO using the calculated values of $F_{L}$ and known parametrized $F_{2}$ structure function.

In Fig.(5) we present the ratio $R$ in comparison with the H1 data at LO up to NNLO approximations. As can be seen in this figure, one can conclude that the NNLO results essentially improve the good agreement with data in comparison with the NLO calculations. The ratio $R$ value determined at NNLO is comparable with those obtained in literature (of the order of 0.1 to 0.3 ).

As in Ref.[48] the ratio $R$ is found at $R=0.260 \pm 0.050$ which this value is constant at the region $7.10^{-5}<x<2.10^{-3}$ and $3.5 \leq Q^{2} \leq 45 \mathrm{GeV}^{2}$. In color dipole model the ratio $R$ lead to the bound $R \leq 0.372$ [49]. In Ref.[50] ZEUS Collaboration is shown that the overall value of $R$ from both the unconstrained and constrained fits is $R=0.105_{-0.037}^{+0.055}$ in wide range of $Q^{2}$ values $\left(5 \leq Q^{2} \leq 110 \mathrm{GeV}^{2}\right)$.

In Fig.(6), the ratio $R$ is plotted against $x$ for different values of $Q^{2}$ in comparison with the $\mathrm{H} 1$ data. We have analyzed the behavior of the ratio $R$ for $Q^{2}$ values of $Q^{2}=5,15,25$ and $45 \mathrm{GeV}^{2}$ in the range $10^{-5}<x<10^{-2}$ which show a good agreement with the H1 data. We observe that the ratio $R$ is consistent with a constant behavior with respect to $x$ for fixed values of $Q^{2}$ at NNLO approximation.

To emphasize the size of the NNLO corrections, we show the ratio NNLO/NLO for the longitudinal structure function $F_{L}$ and the ratio $R$ in Figs.(7) and (8). As can be seen, these corrections are determined in the interval $1 \mathrm{GeV}^{2}<Q^{2}<5000 \mathrm{GeV}^{2}$ and for $Q^{2}=5,15,25$ and $45 \mathrm{GeV}^{2}$ respectively. In Fig.(7) the results for higher values of $Q^{2}\left(Q^{2}>300 \mathrm{GeV}^{2}\right)$ are very similar. It is seen that the ratio NNLO/NLO for the longitudinal structure function $F_{L}$ and the ratio $R$ has not a continuous increase towards large $Q^{2}$ values. In this region a depletion is observable, as the NNLO distributions are smaller in contrast with the NLO distributions. A detailed comparison for the size of the NNLO corrections at four $Q^{2}$ values has been shown in Fig.8. It is observable that the corrections at very low values of $x$ are larger than to the moderate $x$ values.

\section{Summary and Conclusion}

We presented a certain theoretical model to describe the longitudinal structure function data based on the DGLAP evolution equations at low values of $x$ in the leading-order up to next-to-next-to-leading order. The longitudinal structure functions are arising from the coupled DGLAP and Altarelli- Martinelli equations by using the hard-pomeron behavior of the distribution functions. The direct extraction of $F_{L}$ from experimental data is a cumbersome procedure, therefore the possibility 
of the non-direct determination of $F_{L}$ provided by these equations. This method can be used in very low $x$ at the LHeC project. Our results at NNLO are in good agreement with the experimental data at low $x$ values in a large interval of the momentum transfer. The obtained explicit expression for the longitudinal structure function is entirely determined by the effective exponents of the singlet and gluon distribution functions. We have also calculated the ratio $F_{L} / F_{2}$ and the cross section ratio $R$ which indicate a good agreement with the $\mathrm{H} 1$ data at the NNLO approximation. The variations of the ratio $F_{L} / F_{2}$ and $R$ with low values of $x$ and fixed $Q^{2}$ value show a constant behavior similar to that of the experimental data and the color dipole model. Consequently, the size of the NNLO corrections become possible to perform the high-order corrections to the ultra-high energy processes.

\section{ACKNOWLEDGMENTS}

The authors are thankful to the Razi University for financial support of this project.

\section{Appendix A}

The coefficient functions for $F_{L}$ have the following forms [7] as:

At LO :

$$
\begin{aligned}
c_{L, g}^{1} & =8 n_{f} z(1-z), \\
c_{L, q}^{1} & =4 C_{F} z .
\end{aligned}
$$

At NLO :

$$
\begin{aligned}
c_{L, g}^{2}= & n_{f}\left\{(94.74-49.20 z) z_{1} L_{1}^{2}+864.8 z_{1} L_{1}\right. \\
& +1161 z L_{1} L_{0}+60.06 z L_{0}^{2}+39.66 z_{1} L_{0} \\
& -5.333(1 / z-1)\}, \\
c_{L, q}^{2}= & 128 / 9 z L_{1}^{2}-46.50 z L_{1}-84.094 L_{0} L_{1}-37.338 \\
& +89.53 z+33.82 z^{2}+z L_{0}\left(32.90+18.41 L_{0}\right) \\
& -128 / 9 L_{0}-0.012 \delta\left(z_{1}\right)+16 / 27 n_{f}\left(6 z L_{1}\right. \\
& \left.-12 z L_{0}-25 z+6\right) \\
& +n_{f}\left\{(15.94-5.212 z) z_{1}^{2} L_{1}+(0.421+1.520 z) L_{0}^{2}\right. \\
& \left.+28.09 z_{1} L_{0}-(2.371 / z-19.27) z_{1}^{3}\right\} .
\end{aligned}
$$

At NNLO :

$$
\begin{aligned}
& c_{L, g}^{3}=n_{f}\left\{\left(144 L_{1}^{4}-47024 / 27 L_{1}^{3}+6319 L_{1}^{2}+53160 L_{1}\right) z_{1}\right. \\
& +72549 L_{0} L_{1}+88238 L_{0}^{2} L_{1}+(3709-33514 z \\
& \left.-9533 z^{2}\right) z_{1}+66773 z L_{0}^{2}-1117 L_{0}+45.37 L_{0}^{2} \\
& \left.-5360 / 27 L_{0}^{3}-\left(2044.70 z_{1}+409.506 L_{0}\right) 1 / z\right\} \\
& +n_{f}^{2}\left\{\left(32 / 3 L_{1}^{3}-1216 / 9 L_{1}^{2}-592.3 L_{1}\right.\right. \\
& \left.+1511 z L_{1}\right) z_{1}+311.3 L_{0} L_{1}+14.24 L_{0}^{2} L_{1} \\
& +(577.3-729 z) z_{1}+30.78 z L_{0}^{3}+366 L_{0}+1000 / 9 L_{0}^{2} \\
& \left.+160 / 9 L_{0}^{3}+88.50371 / z z_{1}\right\} \\
& +f l_{11}^{g} n_{f}^{2}\left\{\left(-0.0105 L_{1}^{3}+1.550 L_{1}^{2}+19.72 z L_{1}\right.\right. \\
& \left.-66.745 z+0.615 z^{2}\right) z_{1}+20 / 27 z L_{0}^{4}+(280 / 81 \\
& \left.+2.260 z) z L_{0}^{3}-(15.40-2.201 z) z L_{0}^{2}+2.260 z\right) z L_{0}^{3} \\
& \left.-(71.66-0.121 z) z L_{0}\right\} \text {, } \\
& c_{L, q}^{3}=512 / 27 L_{1}^{4}-177.40 L_{1}^{3}+650.6 L_{1}^{2}-2729 L_{1} \\
& -2220.5-7884 z+4168 z^{2}-\left(844.7 L_{0}\right. \\
& \left.+517.3 L_{1}\right) L_{0} L_{1}+\left(195.6 L_{1}-125.3\right) z_{1} L_{1}^{3} \\
& +208.3 z L_{0}^{3}-1355.7 L_{0}-7456 / 27 L_{0}^{2}-1280 / 81 L_{0}^{3} \\
& +0.113 \delta\left(z_{1}\right)+n_{f}\left\{1024 / 81 L_{1}^{3}-112.35 L_{1}^{2}+344.1 L_{1}\right. \\
& +408.4-9.345 z-919.3 z^{2}+\left(239.7+20.63 L_{1}\right) z_{1} L_{1}^{2} \\
& +\left(887.3+294.5 L_{0}-59.14 L_{1}\right) L_{0} L_{1}-1792 / 81 z L_{0}^{3} \\
& \left.+200.73 L_{0}+64 / 3 L_{0}^{2}+0.006 \delta\left(z_{1}\right)\right\}+n_{f}^{2}\left\{3 z L_{1}^{2}\right. \\
& +(6-25 z) L_{1}-19+\left(317 / 6-12 \zeta_{2}\right) z-6 z L_{0} L_{1} \\
& \left.\left.+6 z L i_{2}(x)\right)+9 z L_{0}^{2}-(6-50 z) L_{0}\right\} 64 / 81 \\
& +f l_{11}^{n s} n_{f}\left\{\left(107+321.05 z-54.62 z^{2}\right) z_{1}-26.717\right. \\
& +9.773 L_{0}+\left(363.8+68.32 L_{0}\right) z L_{0} \\
& \left.-320 / 81 L_{0}^{2}\left(2+L_{0}\right)\right\} z \\
& +n_{f}\left\{\left(1568 / 27 L_{1}^{3}-3968 / 9 L_{1}^{2}+5124 L_{1}\right) z_{1}^{2}\right. \\
& +\left(2184 L_{0}+6059 z_{1}\right) L_{0} L_{1}-(795.6+1036 z) z_{1}^{2} \\
& -143.6 z_{1} L_{0}+2848 / 9 L_{0}^{2}-1600 / 27 L_{0}^{3} \\
& \left.-\left(885.53 z_{1}+182 L_{0}\right) 1 / z z_{1}\right\}+n_{f}^{2}\left\{\left(-32 / 9 L_{1}^{2}\right.\right. \\
& \left.+29.52 L_{1}\right) z_{1}^{2}+\left(35.18 L_{0}+73.06 z_{1}\right) L_{0} L_{1} \\
& -35.24 z L_{0}^{2}-(14.16-69.84 z) z_{1}^{2}-69.41 z_{1} L_{0} \\
& \left.-128 / 9 L_{0}^{2}+40.2391 / z z_{1}^{2}\right\}+f l_{11}^{p s} n_{f}\{(107 \\
& \left.+321.05 z-54.62 z^{2}\right) z_{1}-26.717+9.773 L_{0}+(363.8 \\
& \left.\left.+68.32 L_{0}\right) z L_{0}-320 / 81 L_{0}^{2}\left(2+L_{0}\right)\right\} z \text {. }
\end{aligned}
$$

In these equations we have used the abbreviations $z_{1}=1-z, L_{0}=\ln z$ and $L_{1}=\ln z_{1}$. For the $\mathrm{SU}(\mathrm{N})$ gauge group, we have $C_{A}=N, C_{F}=\left(N^{2}-1\right) / 2 N$, $T_{F}=n_{f} T_{R}$, and $T_{R}=1 / 2$ where $C_{F}$ and $C_{A}$ are the color Cassimir operators in QCD. Also the new charge factors are defined by the following form, $f l_{11}^{n s}=3<e>$, $f l_{11}^{g}=<e>^{2} /<e^{2}>$ and $f l_{11}^{p s}=f l_{11}^{g}-f l_{11}^{n s}$. 


\section{Appendix B}

The LO up to NNLO splitting functions for singlet and gluon distribution functions are as follows [29-30]: At LO :

$$
\begin{aligned}
& P_{q q}^{\mathrm{LO}}(z)=C_{F}\left[\frac{1+z^{2}}{(1-z)_{+}}+\frac{3}{2} \delta(1-z)\right] \\
& P_{q g}^{\mathrm{LO}}(z)=\frac{1}{2}\left(z^{2}+(1-z)^{2}\right) \\
& P_{g q}^{\mathrm{LO}}(z)=C_{F} \frac{1+(1-z)^{2}}{z} \\
& P_{g g}^{\mathrm{LO}}(z)=2 C_{A}\left(\frac{z}{(1-z)_{+}}+\frac{(1-z)}{z}+z(1-z)\right) \\
& +\delta(1-z) \frac{\left(11 C_{A}-4 n_{f} T_{R}\right)}{6} .
\end{aligned}
$$

The convolution integrals which contains plus prescription, ()$_{+}$, can be easily calculate by

$$
\begin{aligned}
\int_{x}^{1} \frac{d y}{y} f\left(\frac{x}{y}\right)+g(y)= & \int_{x}^{1} \frac{d y}{y} f\left(\frac{x}{y}\right)\left[g(y)-\frac{x}{y} g(x)\right] \\
& -g(x) \int_{0}^{x} f(y) d y
\end{aligned}
$$

\section{At NLO :}

$$
\begin{aligned}
P_{q q}^{\mathrm{NLO}}= & \left(C_{F}\right)^{2}\left(-1+z+(1 / 2-3 / 2 z) \ln (z)-1 / 2(1+z) \ln (z)^{2}-(3 / 2 \ln (z)+2 \ln (z) \ln (1-z)) p_{q q}(z)\right. \\
& \left.+2 p_{q q}(-z) S_{2}(z)\right)+C_{F} C_{A}\left(14 / 3(1-z)+\left(11 / 6 \ln (z)+1 / 2 \ln (z)^{2}+67 / 18-\pi^{2} / 6\right) p_{q q}(z)\right. \\
& \left.-p_{q q}(-z) S_{2}(z)\right)+C_{F} T_{F}\left(-16 / 3+40 / 3 z+\left(10 z+16 / 3 z^{2}+2\right) \ln (z)-112 / 9 z^{2}+40 /(9 z)\right. \\
& \left.-2(1+z) \ln (z)^{2}-(10 / 9+2 / 3 \ln (z)) p_{q q}(z)\right) \\
P_{q g}^{\mathrm{NLO}=} & C_{F} T_{F}\left(4-9 z-(1-4 z) \ln (z)-(1-2 z) \ln (z)^{2}+4 \ln (1-z)+\left(2 \ln ((1-z) / z)^{2}-4 \ln ((1-z) / z)\right.\right. \\
& \left.\left.-2 / 3 \pi^{2}+10\right) P_{q g}(z)\right)+C_{A} T_{F}(182 / 9+14 / 9 z+40 /(9 z)+(136 / 3 z-38 / 3) \ln (z)-4 \ln (1-z) \\
& -(2+8 z) \ln (z)^{2}+2 P_{q g}(-z) S_{2}(z)+\left(-\ln (z)^{2}+44 / 3 \ln (z)-2 \ln (1-z)^{2}+4 \ln (1-z)\right. \\
& \left.\left.+\pi^{2} / 3-218 / 9\right) P_{q g}(z)\right) \\
= & C_{F}^{2}\left(-5 / 2-7 z / 2+(2+7 / 2 z) \ln (z)-(1-z / 2) \ln (z)^{2}-2 z \ln (1-z)-(3 \ln (1-z)\right. \\
& \left.\left.+\ln (1-z)^{2}\right) P_{g q}(z)\right)+C_{F} C_{A}\left(28 / 9+65 / 18 z+44 / 9 z^{2}-\left(12+5 z+8 / 3 z^{2}\right) \ln (z)+(4+z) \ln (z)^{2}\right. \\
& +2 z \ln (1-z)+S_{2}(z) P_{g q}(-z)+\left(1 / 2-2 \ln (z) \ln (1-z)+1 / 2 \ln (z)^{2}+11 / 3 \ln (1-z)+\ln (1-z)^{2}\right. \\
& \left.\left.-\pi^{2} / 6\right) P_{g q}(z)\right)+C_{F} T_{F}\left(-4 / 3 z-(20 / 9+4 / 3 \ln (1-z)) P_{g q}(z)\right) . \\
& C_{F} T_{F}\left(-16+8 z+20 / 3 z^{2}+4 /(3 z)-(6+10 z) \ln (z)-(2+2 z) \ln (z)^{2}\right)+C_{A} T_{F}(2-2 z \\
& \left.+26 / 9\left(z^{2}-1 / z\right)-4 / 3(1+z) \ln (z)-20 / 9 P_{g g}(z)\right)+C_{A}^{2}\left(27 / 2(1-z)+26 / 9\left(z^{2}-1 / z\right)\right. \\
& -\left(25 / 3-11 / 3 z+44 / 3 z^{2}\right) \ln (z)+4(1+z) \ln (z)^{2}+2 P_{g g}(-z) S_{2}(z)+(67 / 9-4 \ln (z) \ln (1-z) \\
& \left.\left.+\ln (z)^{2}-\pi^{2} / 3\right) P_{g g}(z)\right) .
\end{aligned}
$$

where

\section{At NNLO :}

$$
\begin{array}{r}
p_{q q}(z)=2 /(1-z)-1-z \\
p_{q q}(-z)=2 /(1+z)-1+z \\
P_{q g}(z)=z^{2}+(1-z)^{2} \\
P_{q g}(-z)=z^{2}+(1+z)^{2} \\
P_{g q}(z)=\left(1+(1-z)^{2}\right) / z \\
P_{g q}(-z)=-\left(1+(1+z)^{2}\right) / z \\
P_{g g}(z)=1 /(1-z)+1 / z-2+z(1-z) \\
P_{g g}(-z)=1 /(1+z)-1 / z-2-z(1+z) \\
S_{2}(z)=\int_{1 /(1+z)}^{z /(1+z)} 1 / y \ln ((1-y) / y) d y
\end{array}
$$




$$
\begin{aligned}
& P_{q q}^{\mathrm{NNLO}}=\left(n _ { f } \left(-5.926 L_{1}^{3}-9.751 L_{1}^{2}-72.11 L_{1}+177.4+392.9 z-101.4 z^{2}-57.04 L_{0} L_{1}-661.6 L_{0}\right.\right. \\
& \left.+131.4 L_{0}^{2}-400 / 9 L_{0}^{3}+160 / 27 L_{0}^{4}-506 / z-3584 / 271 / z L_{0}\right)+n_{f}^{2}\left(1.778 L_{1}^{2}+5.944 L_{1}\right. \\
& +100.1-125.2 z+49.26 z^{2}-12.59 z^{3}-1.889 L_{0} L_{1}+61.75 L_{0}+17.89 L_{0}^{2}+32 / 27 L_{0}^{3} \\
& +256 / 811 / z))(1-z) \text {. } \\
& P_{q g}^{\mathrm{NNLO}}=n_{f}\left(100 / 27 L_{1}^{4}-70 / 9 L_{1}^{3}-120.5 L_{1}^{2}+104.42 L_{1}+2522-3316 z+2126 z^{2}\right. \\
& +L_{0} L_{1}\left(1823-25.22 L_{0}\right)-252.5 z L_{0}^{3}+424.9 L_{0}+881.5 L_{0}^{2}-44 / 3 L_{0}^{3}+536 / 27 L_{0}^{4} \\
& \left.-1268.31 / z-896 / 31 / z L_{0}\right)+n_{f}^{2}\left(20 / 27 L_{1}^{3}+200 / 27 L_{1}^{2}-5.496 L_{1}-252+158 z+145.4 z^{2}\right. \\
& -139.28 z^{3}-L_{0} L_{1}\left(53.09+80.616 L_{0}\right)-98.07 z L_{0}^{2}+11.70 z L_{0}^{3}-254 L_{0}-98.80 L_{0}^{2}-376 / 27 L_{0}^{3} \\
& \left.-16 / 9 L_{0}^{4}+1112 / 2431 / z\right) \text {. } \\
& P_{g q}^{\mathrm{NNLO}}=400 / 81 L_{1}^{4}+2200 / 27 L_{1}^{3}+606.3 L_{1}^{2}+2193 L_{1}-4307+489.3 z+1452 z^{2}+146 z^{3}-447.3 L_{0}^{2} L_{1} \\
& -972.9 z L_{0}^{2}+4033 L_{0}-1794 L_{0}^{2}+1568 / 9 L_{0}^{3}-4288 / 81 L_{0}^{4}+6163.11 / z+1189.31 / z L_{0} \\
& +n_{f}\left(-400 / 81 L_{1}^{3}-68.069 L_{1}^{2}-296.7 L_{1}-183.8+33.35 z-277.9 z^{2}+108.6 z L_{0}^{2}\right. \\
& \left.-49.68 L_{0} L_{1}+174.8 L_{0}+20.39 L_{0}^{2}+704 / 81 L_{0}^{3}+128 / 27 L_{0}^{4}-46.411 / z+71.0821 / z L_{0}\right) \\
& +n_{f}^{2}\left(96 / 27 L_{1}^{2}(1 / z-1+1 / 2 z)+320 / 27 L_{1}(1 / z-1+4 / 5 z)-64 / 27(1 / z-1-2 z)\right) \text {. } \\
& P_{g g}^{\mathrm{NNLO}}=2643.524 D_{0}+4425.894 \delta(1-z)+3589 L_{1}-20852+3968 z-3363 z^{2}+4848 z^{3} \\
& +L_{0} L_{1}\left(7305+8757 L_{0}\right)+274.4 L_{0}-7471 L_{0}^{2}+72 L_{0}^{3}-144 L_{0}^{4}+142141 / z+2675.81 / z L_{0} \\
& +n_{f}\left(-412.142 D_{0}-528.723 \delta(1-z)-320 L_{1}-350.2+755.7 z-713.8 z^{2}+559.3 z^{3}\right. \\
& +L_{0} L_{1}\left(26.15-808.7 L_{0}\right)+1541 L_{0}+491.3 L_{0}^{2}+832 / 9 L_{0}^{3}+512 / 27 L_{0}^{4}+182.961 / z \\
& \left.+157.271 / z L_{0}\right)+n_{f}^{2}\left(-16 / 9 D_{0}+6.4630 \delta(1-z)-13.878+153.4 z-187.7 z^{2}+52.75 z^{3}\right. \\
& \left.-L_{0} L_{1}\left(115.6-85.25 z+63.23 L_{0}\right)-3.422 L_{0}+9.680 L_{0}^{2}-32 / 27 L_{0}^{3}-680 / 2431 / z\right) .
\end{aligned}
$$

where $D_{0}=1 /(1-z)$.

\section{Appendix C}

We present here the kernels for the quark and gluon sectors, denoted by $\Phi$ and $\Theta$ respectively at LO up to NNLO which we used in Eqs.(19-21).

$$
\begin{aligned}
\Theta_{g q}\left(x, Q^{2}\right) & =P_{g q}\left(x, \alpha_{s}\right) \odot x^{\lambda_{s}}, \\
\Theta_{q g}\left(x, Q^{2}\right) & =P_{q g}\left(x, \alpha_{s}\right) \odot x^{\lambda_{g}}, \\
\Phi_{g g}\left(x, Q^{2}\right) & =P_{g g}\left(x, \alpha_{s}\right) \odot x^{\lambda_{g}}, \\
\Phi_{q q}\left(x, Q^{2}\right) & =P_{q q}\left(x, \alpha_{s}\right) \odot x^{\lambda_{s}},
\end{aligned}
$$

where the splitting functions expanded into one, two and three loops correction in accordance with Appendix B. As the required leading order approximation of the singlet kernels are written as:

$$
\begin{aligned}
\Phi_{q q}\left(x, Q^{2}\right)= & \frac{\alpha_{s}}{4 \pi}\left\{4+\frac{16}{3} \ln \left(\frac{1-x}{x}\right)+\frac{16}{3} \int_{x}^{1} \frac{z^{\lambda_{s}}-z^{-1}}{1-z} d z\right. \\
& \left.-\frac{8}{3} \int_{x}^{1}(1+z) z^{\lambda_{s}} d z\right\} \\
\Theta_{q g}\left(x, Q^{2}\right)= & \frac{\alpha_{s}}{4 \pi} \frac{20}{9} \int_{x}^{1}\left(z^{2}+(1-z)^{2}\right) z^{\lambda_{g}} d z
\end{aligned}
$$

The longitudinal kernels at low- $x$ limit are given by

$$
\begin{aligned}
& I_{L, q}\left(x, Q^{2}\right)=C_{L, q}\left(x, \alpha_{s}\right) \odot x^{\lambda_{s}}, \\
& I_{L, g}\left(x, Q^{2}\right)=C_{L, g}\left(x, \alpha_{s}\right) \odot x^{\lambda_{g}} .
\end{aligned}
$$

Here the longitudinal coefficient functions $C_{L, a}\left(x, \alpha_{s}\right), \quad a=q, \quad g$ are the singlet and gluon functions known perturbatively upto first few orders in the running coupling constant $\alpha_{s}$ and can be written as

$$
\begin{aligned}
C_{L, a}\left(x, \alpha_{s}\right)= & \frac{\alpha_{s}}{4 \pi} c_{L, a}^{1}(x)+\left(\frac{\alpha_{s}}{4 \pi}\right)^{2} c_{L, a}^{2}(x) \\
& +\left(\frac{\alpha_{s}}{4 \pi}\right)^{3} c_{L, a}^{3}(x),
\end{aligned}
$$

where $c_{L, a}(x)$ are given in appendix A. The required leading order approximation of the longitudinal singlet and 
gluon coefficient functions are written as:

$$
\begin{aligned}
& I_{L, q}\left(x, Q^{2}\right)=\frac{\alpha_{s}}{4 \pi} \int_{x}^{1} 8 n_{f}(1-z) z^{\lambda_{s}+1} d z \\
& I_{L, g}\left(x, Q^{2}\right)=\frac{\alpha_{s}}{4 \pi} \int_{x}^{1} 4 C_{F} z^{\lambda_{g}+1} d z .
\end{aligned}
$$

\section{Appendix D}

The proton structure function parameterized with a global fit function [39] to the HERA combined data for $F_{2}^{\gamma p}\left(x, Q^{2}\right)$ for $0.85<Q^{2}<3000 \mathrm{GeV}^{2}$ and $x<0.1$, which ensures that the saturated Froissart $\ln ^{2}(1 / x)$ behavior dominates at low- $x$. This global fit takes the form

$$
\begin{aligned}
F_{2}^{\gamma p}\left(x, Q^{2}\right)= & (1-x)\left[\frac{F_{P}}{1-x_{P}}+A\left(Q^{2}\right) \ln \left(\frac{x_{P}}{x} \frac{1-x}{1-x_{P}}\right)\right. \\
& \left.+B\left(Q^{2}\right) \ln ^{2}\left(\frac{x_{P}}{x} \frac{1-x}{1-x_{P}}\right)\right],
\end{aligned}
$$

where

$$
A\left(Q^{2}\right)=a_{0}+a_{1} \ln Q^{2}+a_{2} \ln ^{2} Q^{2},
$$

and

$$
B\left(Q^{2}\right)=b_{0}+b_{1} \ln Q^{2}+b_{2} \ln ^{2} Q^{2} .
$$

The fitted parameters are tabulated in Table I. At low $x$ ( or large $\nu=\ln (1 / x))$, the global fit becomes a quadratic polynomial in $\nu$ as

$$
\widehat{F}_{2}^{\gamma p}\left(\nu, Q^{2}\right) \rightarrow C_{0 f}\left(Q^{2}\right)+C_{1 f}\left(Q^{2}\right) \nu+C_{2 f}\left(Q^{2}\right) \nu^{2}+\widehat{O}(\nu)
$$
where the coefficient functions are defined in Ref. [39].

TABLE I: Parameters of Eq. (41), resulting from a global fit to the HERA combined data.

\begin{tabular}{cc}
\hline \hline parameters & value \\
& \\
$a_{0}$ & $-8.471 \times 10^{-2} \pm 2.62 \times 10^{-3}$ \\
$a_{1}$ & $4.190 \times 10^{-2} \pm 1.56 \times 10^{-3}$ \\
$a_{2}$ & $-3.976 \times 10^{-3} \pm 2.13 \times 10^{-4}$ \\
$b_{0}$ & $1.292 \times 10^{-2} \pm 3.62 \times 10^{-4}$ \\
$b_{1}$ & $2.473 \times 10^{-4} \pm 2.46 \times 10^{-4}$ \\
$b_{2}$ & $1.642 \times 10^{-3} \pm 5.52 \times 10^{-5}$ \\
$F_{p}$ & $0.413 \pm 0.003$ \\
$\chi^{2}$ (goodness of fit) & 1.17 \\
\hline
\end{tabular}

This form for $F_{2}^{\gamma p}$ (i.e.Eq.(41)) describes the HERA data well, but the model does not have the properties necessary for the $\gamma^{*}-p$ reduced cross section to extend smoothly to $Q^{2}=0$ limit. Authors in Ref.[19] provide good fits to the HERA data at low $x$ and large $Q^{2}$ values. With respect to the Block-Halzen [40] fit, The explicit expression for the proton structure function in a range of the kinematical variables $x$ and $Q^{2}, x<0.001$ and $0.15 \mathrm{GeV}^{2}<Q^{2}<3000 \mathrm{GeV}^{2}$, is defined by the following form

$$
\begin{aligned}
F_{2}^{\gamma p}\left(x, Q^{2}\right)= & D\left(Q^{2}\right)(1-x)^{n}\left[C\left(Q^{2}\right)+A\left(Q^{2}\right) \ln \left(\frac{1}{x} \frac{Q^{2}}{Q^{2}+\mu^{2}}\right)\right. \\
& \left.+B\left(Q^{2}\right) \ln ^{2}\left(\frac{1}{x} \frac{Q^{2}}{Q^{2}+\mu^{2}}\right)\right],
\end{aligned}
$$

where

$$
\begin{aligned}
& A\left(Q^{2}\right)=a_{0}+a_{1} \ln \left(1+\frac{Q^{2}}{\mu^{2}}\right)+a_{2} \ln ^{2}\left(1+\frac{Q^{2}}{\mu^{2}}\right), \\
& B\left(Q^{2}\right)=b_{0}+b_{1} \ln \left(1+\frac{Q^{2}}{\mu^{2}}\right)+b_{2} \ln ^{2}\left(1+\frac{Q^{2}}{\mu^{2}}\right), \\
& C\left(Q^{2}\right)=c_{0}+c_{1} \ln \left(1+\frac{Q^{2}}{\mu^{2}}\right), \\
& D\left(Q^{2}\right)=\frac{Q^{2}\left(Q^{2}+\lambda M^{2}\right)}{\left(Q^{2}+M^{2}\right)^{2}} .
\end{aligned}
$$

Here $M$ is the effective mass and $\mu^{2}$ is a scale factor. The additional parameters with their statistical errors are given in Table II.

TABLE II: The effective Parameters at low $x$ for $0.15 \mathrm{GeV}^{2}<$ $Q^{2}<3000 \mathrm{GeV}^{2}$ provided by the following values. The fixed parameters are defined by the Block-Halzen fit to the real photon-proton cross section as $M^{2}=0.753 \pm 0.068 \mathrm{GeV}^{2}$, $\mu^{2}=2.82 \pm 0.290 \mathrm{GeV}^{2}$ and $c_{0}=0.255 \pm 0.016$ [19]. value

\begin{tabular}{cc}
$a_{0}$ & $8.205 \times 10^{-4} \pm 4.62 \times 10^{-4}$ \\
$a_{1}$ & $-5.148 \times 10^{-2} \pm 8.19 \times 10^{-3}$ \\
$a_{2}$ & $-4.725 \times 10^{-3} \pm 1.01 \times 10^{-3}$ \\
$b_{0}$ & $2.217 \times 10^{-3} \pm 1.42 \times 10^{-4}$ \\
$b_{1}$ & $1.244 \times 10^{-2} \pm 8.56 \times 10^{-4}$ \\
$b_{2}$ & $5.958 \times 10^{-4} \pm 2.32 \times 10^{-4}$ \\
& \\
$C_{1}$ & $1.475 \times 10^{-1} \pm 3.025 \times 10^{-2}$ \\
$n$ & $11.49 \pm 0.99$ \\
$\lambda$ & $2.430 \pm 0.153$ \\
$\chi^{2}$ (goodness of fit) & 0.95 \\
\hline
\end{tabular}


TABLE III: The QCD coupling and corresponding $\Lambda$ parameter for $n_{f}=4$ at LO, NLO [19, 43] and NNLO analysis [44].

\begin{tabular}{l|c|c|}
\hline & $\alpha_{s}\left(M_{Z}^{2}\right)$ & $\Lambda_{Q C D}(\mathrm{MeV})$ \\
\hline LO & 0.1166 & 136.8 \\
NLO & 0.1166 & 284 \\
NNLO & 0.1155 & 235 \\
\hline
\end{tabular}

\section{REFERENCES}

1. G.G.Callan and D.Gross, Phys.Lett.B22, $156(1969)$.

2. L.A.Anchordoqui, C.Garca Canal and J.F.Soriano, arXiv:1902.10134 hep-ph].

3. M. Klein, Ann. Phys.528, 138 (2016).

4. P.Monaghan et al., Phys.Rev.Lett.110, 152002(2013).

5. V.Andreev et al. [H1 Collaboration], Eur.Phys.J.C74, 2814 (2014).

6. D.I.Kazakov, et.al., Phys.Rev.Lett. 65, 1535(1990).

7. S.Moch, J.A.M.Vermaseren, A.Vogt, Phys.Lett.B 606, 123(2005).

8. M.Gluk, C.Pisano and E.Reya, Phys.Rev.D77, 074002(2008).

9. A.V.Kotikov and G.Parente, JHEP 85, 17(1997).

10. A.V.Kotikov and G.Parente, Mod.Phys.Lett.A12, 963(1997).

11. G.R.Boroun and B.Rezaei, Eur.Phys.J.C72, 2221(2012).

12. B.Rezaei and G.R.Boroun, Nucl.Phys.A857, 42(2011).

13. G.R.Boroun, B.Rezaei and J.K.Sarma, Int.J.Mod.Phys.A29, 1450189(2014).

14. N.Baruah, N.M. Nath, J.K. Sarma , Int.J.Theor.Phys.52, 2464(2013).

15. G.R.Boroun, Phys.Rev.C97, 015206 (2018); B.Rezaei and G.R.boroun, Phys.Rev.C101, 045202(2020).

16. L.P.Kaptari et al., JETP Lett.109, 281(2019).

17. L.P.Kaptari et al., Phys.Rev.D99, 096019(2019).

18. M.M.Block et al., Phys.Rev.D84, 094010 (2011).

19. M.M.Block et al., Phys.Rev.D89, 094027 (2014).

20. G.Altarelli and G.Martinelli, Phys.Lett.B76, 89(1978).

21.Yu.L.Dokshitzer, Sov.Phys.JETP 46, 641(1977); G.Altarelli and G.Parisi, Nucl.Phys.B 126, 298(1977); V.N.Gribov and L.N.Lipatov, Sov.J.Nucl.Phys. 15, 438(1972).

22. A.Donnachie and P.V.Landshoff, Phys.Lett.B 437, 408(1998).

23. A.Donnachie and P.V.Landshoff, Phys.Lett.B 550, $160(2002)$.

24. B.G. Shaikhatdenov, A.V. Kotikov, V.G. Krivokhizhin, G. Parente, Phys. Rev. D 81, 034008(2010). 25. M.Devee, R.Baishya and J.K.sarma, Eur.Phys.J.C72, 2036(2012); M.M.Block et al.,
Phys.Rev.D83, 054009(2011); M.M.Block et al., Eur.Phys.J.C69, 425 (2010); A.Cafarella et al., Nucl.Phys.B748, 253(2006); A.Cafarella et al., Comput.Phys.Commun.179, 665(2008).

26. G.R.Boroun and B.Rezaei, Eur.Phys.J.C73, 2412(2013); S.Zarrin and G.R.Boroun, Nucl.Phys.B922, 126 (2017); F. Taghavi-Shahri, A. Mirjalili and M.M. Yazdanpanah , Eur.Phys.J.C71, 1590(2011); H.Khanpour et al., Phys.Rev.C95, 035201(2017).

27. E. Floratos, C. Kounnas and R. Lacaze, Nucl. Phys. B 192, 417(1981); J. Blumlein, V. Ravindran and W. van Neerven, Nucl. Phys. B 586, 349(2000).

28. C.G. Callan, Jr., Phys. Rev. D2, 1541 (1970); K. Symanzik, Comm. Math. Phys.18, 227 (1970), Comm. Math. Phys. B39,49 (1971); G. Parisi, Phys. Lett. B39, 643 (1972).

29. W.L. van Neerven, A.Vogt, Phys.Lett.B 490, 111(2000).

30. A.Vogt, S.Moch, J.A.M.Vermaseren, Nucl.Phys.B 691, 129(2004).

31. A.A.Godizov, Phys.Rev.D96, 034023 (2017).

32. M.M.Block et al., Phys.Rev.D77, 094003 (2008).

33. A.D.Martin, W.J.stirling and R.S.Thorne, Phys.Lett.B635, 305(2006).

34. K Golec-Biernat and A.M.Stasto, Phys.Rev.D 80, 014006(2009).

35. B.Rezaei and G.R.boroun, Eur.Phys.J.A55, 66(2019); G.R.Boroun, Eur.Phys.J.Plus 135, 68(2020).

36. N.N.Nikolaev and B.G.Zakharov, Phys.Lett.B332, 184(1994); N.N.Nikolaev and V.R.Zoller, Phys.Atom.Nucl.73, 672 (2010).

37. B.Rezaei and G.R.boroun, Phys.Lett.B692, $247(2010)$.

38. M.Froissart, Phys.Rev.123, 1053 (1961).

39. M.M.Block et al., Phys.Rev.D88, 014006 (2013).

40. M. M. Block and F. Halzen, Phys. Rev. D70, 091901(2004).

41. F. D. Aaron et al. [H1 and ZEUS Collaborations], JHEP1001, 109 (2010).

42. A. Y. Illarionov, A. V. Kotikov and G. Parente Bermudez, Phys. Part. Nucl. 39, 307 (2008).

43. S. Chekanov et al. [ZEUS Collaboration], Eur. Phys. J. C21, 443 (2001).

44. A.D.Martin et al., Phys.Letts.B604, 61(2004).

45. M.Niedziela and M.Praszalowicz, Acta Phys.Polon. B46, 2019(2015).

46. C.Ewerz et al., Phys.lett.B720, 181(2013).

47. V.Tvaskis et al., Phys.Rev.C97, 045204(2018); Phys.Rev.Lett. 98 (2007) 142301.

48. F.D. Aaron et al. [H1 Collaboration], phys.Lett.B665, 139(2008); Eur.Phys.J.C71,1579(2011). 49. C.Ewerz and O.Nachtmann, Phys.Lett.B648, 279(2007).

50. H.Abromowicz et al. [ZEUS Collaboration], Phys.Rev.D9, 072002(2014). 


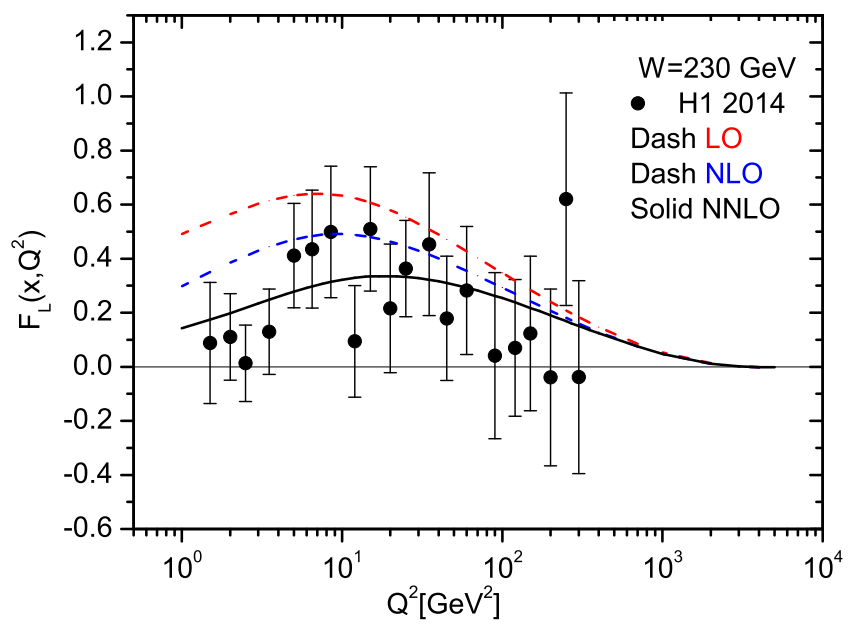

FIG. 1: The longitudinal structure function extracted at LO upto NNLO in comparison with the H1 data [5] as accompanied with total errors. The results are presented at fixed value of the invariant mass $\mathrm{W}$ in the interval $1 \mathrm{GeV}^{2} \leq Q^{2} \leq$ $3000 \mathrm{GeV}^{2}$ at low values of $x$.

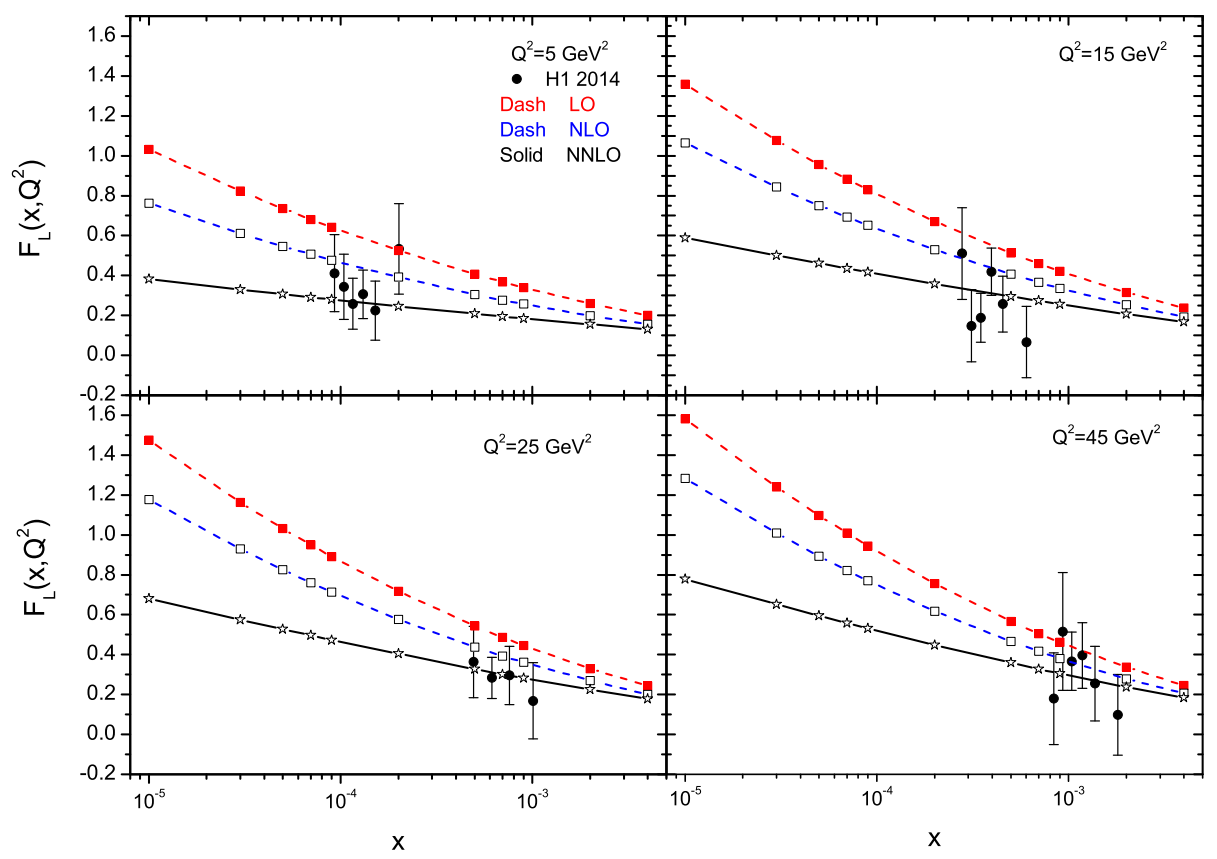

FIG. 2: Predictions of $F_{L}\left(x, Q^{2}\right)$ at four $Q^{2}$ values $5,15,25$ and $45 \mathrm{GeV}^{2}$ at LO upto NNLO, compared with the $\mathrm{H} 1$ data [5]. Dash lines are the longitudinal structure function at LO (full square ) and NLO (open square). Solid line is the longitudinal structure function at NNLO approximation(open star). 


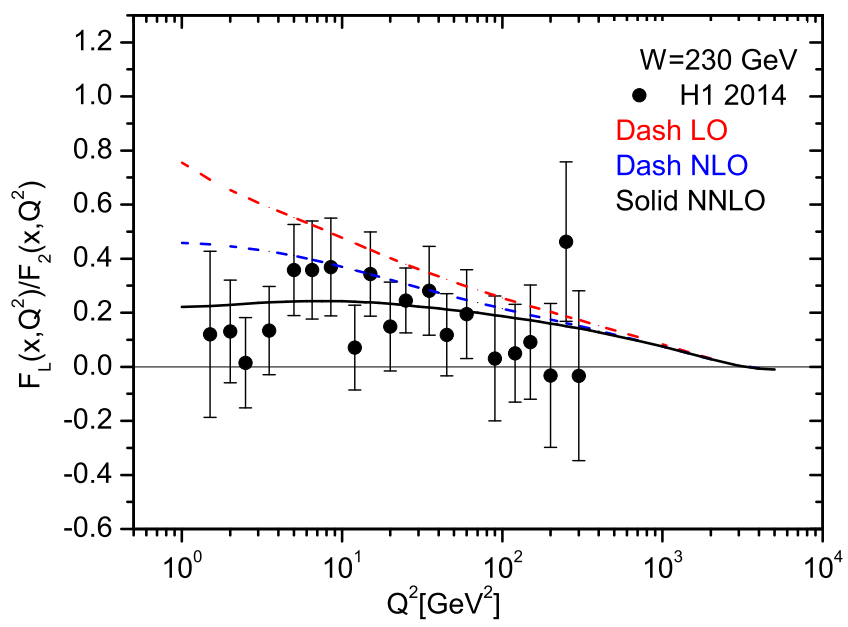

FIG. 3: Ratio $F_{L} / F_{2}$ plotted as function of $Q^{2}$ variable at $W=230 \mathrm{GeV}$ compared with the $\mathrm{H} 1$ data [5].

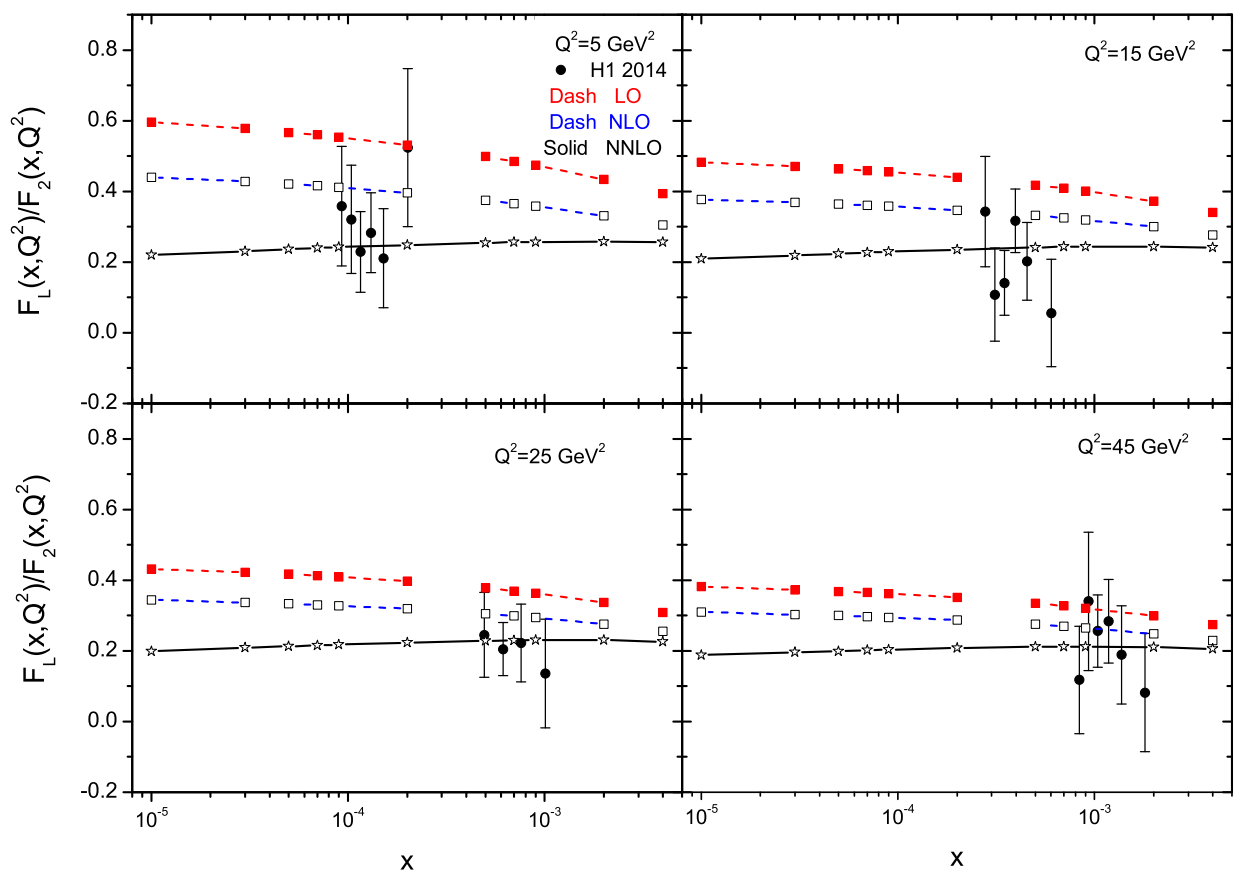

FIG. 4: Predictions of the ratio $F_{L} / F_{2}$ at four $Q^{2}$ values $5,15,25$ and $45 \mathrm{GeV}^{2}$ at LO upto NNLO, compared with the $\mathrm{H} 1$ data [5]. Dash lines are the longitudinal structure function at LO (full square ) and NLO (open square). Solid line is the longitudinal structure function at NNLO approximation(open star). 


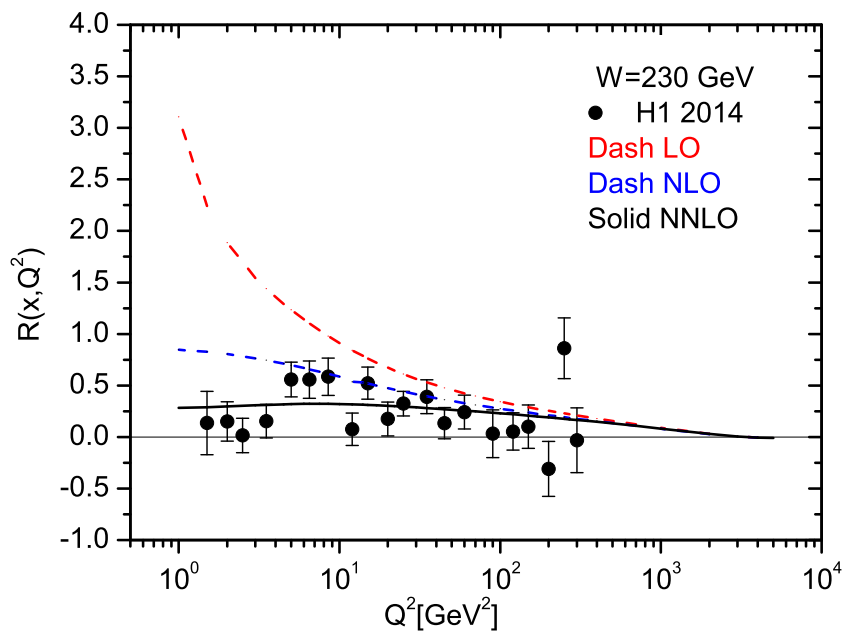

FIG. 5: Ratio $R\left(x, Q^{2}\right)$ plotted as function of $Q^{2}$ variable at $W=230 \mathrm{GeV}$ compared with the $\mathrm{H} 1$ data [5].

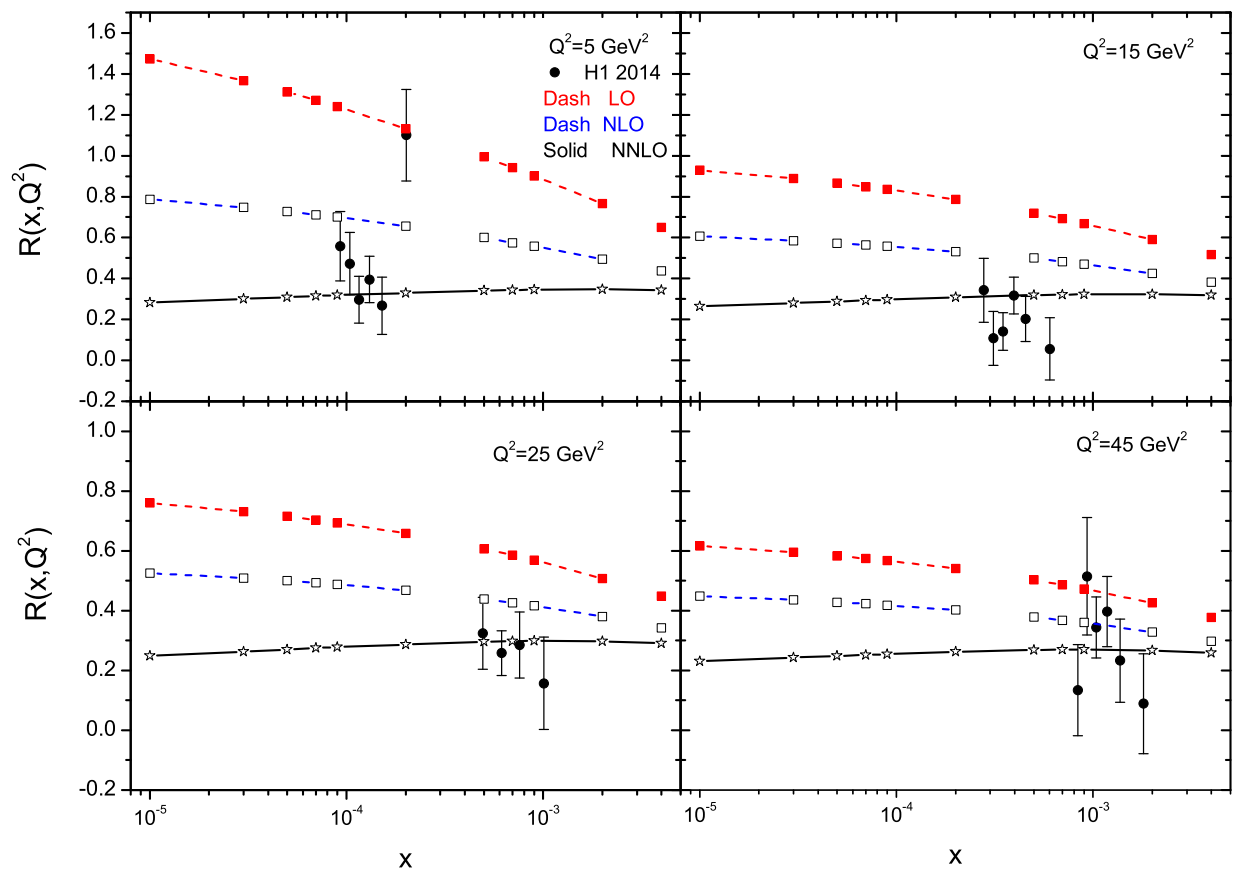

FIG. 6: Predictions of the ratio $R\left(x, Q^{2}\right)$ at four $Q^{2}$ values $5,15,25$ and $45 \mathrm{GeV}^{2}$ at LO upto NNLO, compared with the $\mathrm{H} 1$ data [5]. Dash lines are the longitudinal structure function at LO (full square ) and NLO (open square). Solid line is the longitudinal structure function at NNLO approximation (open star). 


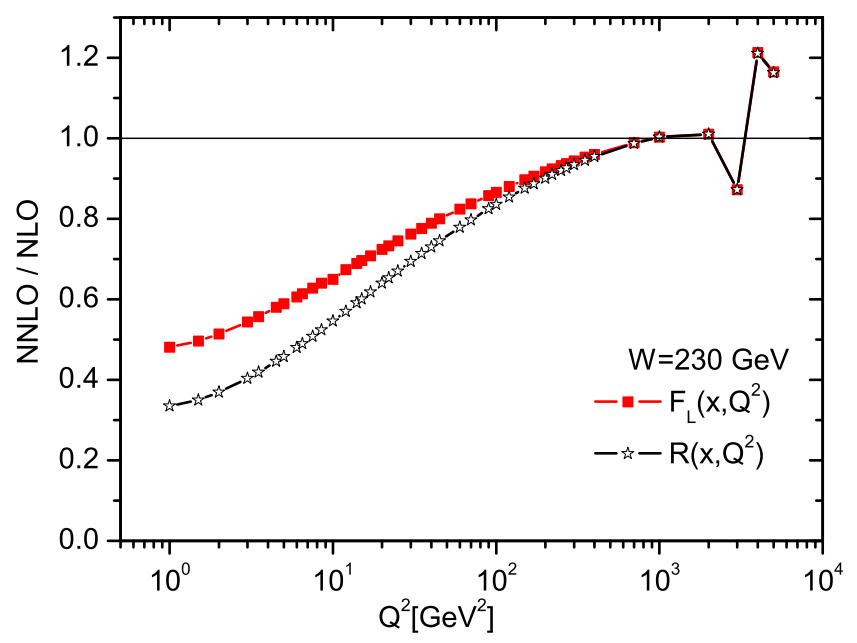

FIG. 7: The NNLO correction factor for $F_{L}\left(x, Q^{2}\right)$ (full square) and $R\left(x, Q^{2}\right)$ (open star) in the interval $1 \mathrm{GeV}^{2}<$ $Q^{2}<5000 \mathrm{GeV}^{2}$ at fixed value of the invariant mass $W=$ $230 \mathrm{GeV}$.

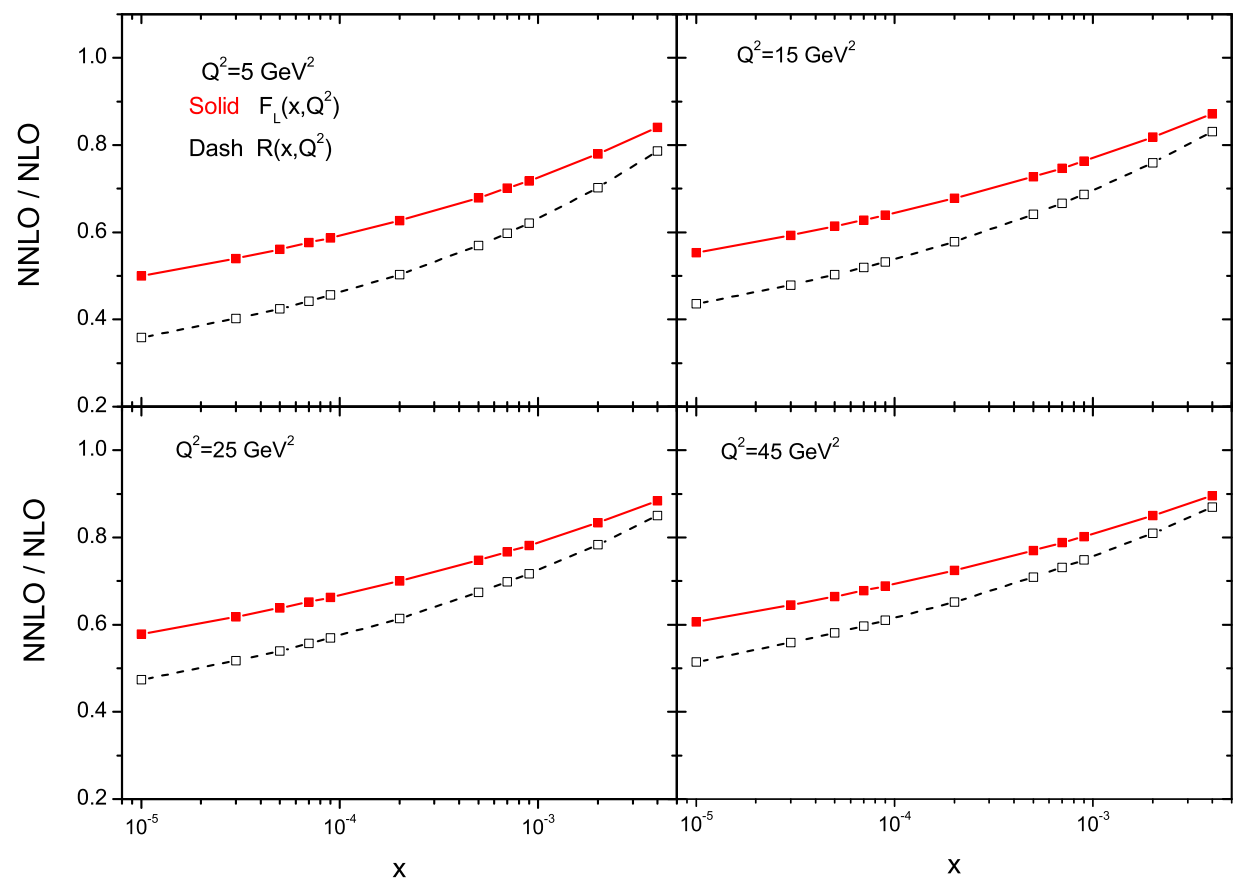

FIG. 8: Ratio of the NNLO to NLO contributions to $F_{L}\left(x, Q^{2}\right)$ (full square) and $R\left(x, Q^{2}\right)$ (open square) for $Q^{2}=$ $5,15,25$ and $45 \mathrm{GeV}^{2}$. 